\title{
Short communication: Improving the accuracy of genomic prediction of body conformation traits in Chinese Holsteins using markers derived from high-density marker panels
}

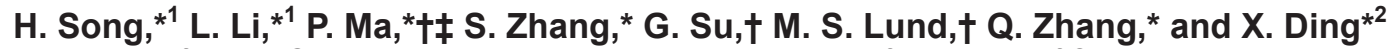 \\ *Laboratory of Animal Genetics, Breeding and Reproduction, Ministry of Agriculture of China, National Engineering Laboratory \\ for Animal Breeding, College of Animal Science and Technology, China Agricultural University, Beijing 100193, P.R. China \\ †Department of Molecular Biology and Genetics, Aarhus University, DK-8830 Tjele, Denmark \\ fDepartment of Animal Science, School of Agriculture and Biology, Shanghai Jiao Tong University, Shanghai 200240, P.R. China
}

\section{ABSTRACT}

This study investigated the efficiency of genomic prediction with adding the markers identified by genomewide association study (GWAS) using a data set of imputed high-density (HD) markers from $54 \mathrm{~K}$ markers in Chinese Holsteins. Among 3,056 Chinese Holsteins with imputed HD data, 2,401 individuals born before October 1, 2009, were used for GWAS and a reference population for genomic prediction, and the 220 younger cows were used as a validation population. In total, $1,403,1,536$, and 1,383 significant single nucleotide polymorphisms (SNP; false discovery rate at 0.05) associated with conformation final score, mammary system, and feet and legs were identified, respectively. About 2 to $3 \%$ genetic variance of 3 traits was explained by these significant SNP. Only a very small proportion of significant SNP identified by GWAS was included in the $54 \mathrm{~K}$ marker panel. Three new marker sets $(54 \mathrm{~K}+)$ were herein produced by adding significant SNP obtained by linear mixed model for each trait into the $54 \mathrm{~K}$ marker panel. Genomic breeding values were predicted using a Bayesian variable selection (BVS) model. The accuracies of genomic breeding value by BVS based on the $54 \mathrm{~K}+$ data were 2.0 to $5.2 \%$ higher than those based on the $54 \mathrm{~K}$ data. The imputed HD markers yielded $1.4 \%$ higher accuracy on average (BVS) than the $54 \mathrm{~K}$ data. Both the $54 \mathrm{~K}+$ and $\mathrm{HD}$ data generated lower bias of genomic prediction, and the $54 \mathrm{~K}+$ data yielded the lowest bias in all situations. Our results show that the imputed HD data were not very useful for improving the accuracy of genomic prediction and that adding the significant markers derived from the imputed HD marker panel could improve the accuracy of genomic prediction and decrease the bias of genomic prediction.

Received July 7, 2017.

Accepted November 25, 2017.

${ }^{1}$ These authors contributed equally to this work.

${ }^{2}$ Corresponding author: xding@cau.edu.cn
Key words: genome-wide association study, genomic prediction, body conformation traits, imputation

\section{Short Communication}

Recently, with the rapid development of technology for high-density (HD) arrays, SNP panels are available for most farm animal species. Several different types of SNP chips with different marker densities have been developed for cattle by Illumina (San Diego, $\mathrm{CA}$ ), including $3 \mathrm{~K}$ (Bovine3K), 7K (BovineLD), 54K ( 54,000 markers; BovineSNP50), and 777K ( 777,000 markers; BovineHD). To date, the $54 \mathrm{~K}$ chip has been widely used in dairy cattle for both genomic selection (Meuwissen et al., 2001) and genome-wide association study (GWAS). Due to the high genotyping cost of BovineHD, it is attractive to impute $54 \mathrm{~K}$ to an HD chip to improve the resolution of GWAS without increasing cost. Several studies reported that genotyping larger numbers of SNP through the use of higher density genotyping arrays can increase the power of the resultant GWAS (Spencer et al., 2009; Meredith et al., 2013; Sahana et al., 2014).

Alternatively, SNP preselection could be efficient to improve the accuracy of genomic prediction (Ober et al., 2012). Several studies reported that incorporating prior knowledge of complex traits can improve the accuracy of genomic prediction (Zhang et al., 2014; Brøndum et al., 2015). Genome-wide association studies have been successfully implemented in a growing list of species, as reviewed by Goddard and Hayes (2009). Conversely, results from GWAS have already been pronounced to be useful for improving genomic prediction.

The objective of this study was to investigate the efficiency of genomic prediction with adding the markers identified by GWAS using a data set of HD markers that were imputed from $54 \mathrm{~K}$ SNP with 3 body conformation traits - conformation final score (BC), mammary system (MS), and feet and legs (FL) - in Chinese Holsteins. 
The data consisted of 3,056 Chinese Holstein cows born from 2000 to 2012. The official EBV on 3 body conformation traits (BC, MS, and FL) were calculated in 2016 by the Dairy Association of China based on a multiple trait random regression test-day model. The resulting reliabilities ranged from 0.5 to 0.6 . To overcome the double counting of conventional EBV as a response variable to genomic prediction, deregressed proof (DRP) was computed based on EBV using the approach proposed by Jairath et al. (1998). The individuals with reliability of DRP $<0.3$ were removed, and 2,621 individuals were retained for further study. Out of the 2,621 Chinese Holsteins, 2,401 cows and bulls born before October 1, 2009, were used for GWAS and taken as the reference population for genomic prediction. The remaining 220 cows born after October 1, 2009, were used as the validation population for genomic prediction.

All individuals with body conformation records were genotyped with the Illumina BovineSNP50 BeadChip. Another 595 bulls (85 Chinese Holsteins and 510 Nordic Holsteins) genotyped with the Illumina BovineSNPHD BeadChip were used as a reference population for imputation. The SNP were filtered with the criteria that the minor allele frequency should be $\geq 0.01$ and that the linkage disequilibrium of adjacent SNP, measured as $r_{\mathrm{LD}}^{2}$ (Hill and Robertson, 1968), should be $\leq 1.0$. In total, 644,400 SNP were retained for imputation. Fimpute (Sargolzaei et al., 2011) was used to impute the 2,621 Chinese Holsteins from 54K to HD. The SNP with a call rate $<0.90$ or a minor allele frequency $<0.03$ were also removed for $54 \mathrm{~K}$ data. In total, $52,886 \mathrm{SNP}$ were retained. After imputation, a quality control procedure was implemented using the following criteria: (1) SNP editing was the same as for $54 \mathrm{~K}$ data, (2) removal of SNP with a $P$-value of Hardy-Weinberg $<10^{-6}$, and (3) removal of individuals with a call rate $<0.90$. After quality control, 578,806 SNP were used for GWAS and genomic prediction.

A linear mixed (LM) model was implemented to identify SNP associated with 3 body conformation traits. The statistical model was

$$
\mathbf{y}=\mathbf{1} \mu+\mathbf{x g}+\mathbf{Z u}+\mathbf{e},
$$

where $\mathbf{y}$ is the vector of DRP; $\mathbf{1}$ is a vector of ones; $\mu$ is the overall mean; $g$ is the average effect of the gene substitution of a particular SNP; $\mathbf{x}$ is a vector of the SNP genotype (coded as 0,1 , or 2 ); $\mathbf{u}$ is the vector of random polygenic effects following the normal distribution $\mathbf{u} \sim N\left(0, \mathbf{G} \sigma_{g}^{2}\right)$, in which $\sigma_{g}^{2}$ is the polygenic variance; $\mathbf{G}$ is the genomic relationship matrix and was constructed using all markers following VanRaden
(2008); $\mathbf{Z}$ is an incidence matrix relating phenotypes to the corresponding random polygenic effects; $\mathbf{e}$ is a vector of residual effects with normal distribution of $N\left(0, \mathbf{I} \sigma_{e}^{2}\right)$, where $\sigma_{e}^{2}$ is the residual variance; and $\mathbf{I}$ is the identity matrix. The software GCTA (Yang et al., 2011) was used to fit the model, and FDR with 0.05 was used for multiple test correction to identify significant SNP.

Three SNP data sets were used to predict genomic breeding values: $54 \mathrm{~K}, 54 \mathrm{~K}+$, and HD. The $54 \mathrm{~K}+$ data set was generated by adding significant SNP identified by GWAS into $54 \mathrm{~K}$ data for each trait. The Bayesian variable selection (BVS) model was implemented to predict genomic EBV (GEBV) for each individual. For the BVS model, all SNP effects were estimated based on the reference population, and the GEBV of a genotyped individual was calculated as the sum of all SNP effects. According to Erbe et al. (2012), the following model was used to estimate the effects of all SNP simultaneously:

$$
\mathbf{y}=\mathbf{1} \mu+\sum_{i=1}^{N} \mathbf{x}_{\mathbf{i}} \mathbf{g}_{\mathrm{i}}+\mathbf{Z u}+\mathbf{e},
$$

where $\mathbf{y}, \mathbf{1}, \mu$, and $\mathbf{e}$ are the same as in the LM model; $\sum_{i=1}^{N} \mathbf{x}_{\mathbf{i}} \mathrm{g}_{\mathrm{i}}$ fits additive SNP effects; $\mathbf{x}_{\mathbf{i}}$ is the vector of SNP genotypes (coded as $0,1,2$ ); and $g_{i}$ is the effect of SNP i, which follows a mixture of normal distribution $\mathrm{g}_{\mathrm{i}} \sim N\left(0, \sigma_{i}^{2}\right)$. The variance of the ith SNP effect had 4 possible values: $\sigma_{1}^{2}=0, \sigma_{2}^{2}=0.0001 \sigma_{g}^{2}, \sigma_{3}^{2}=0.001 \sigma_{g}^{2}$, and $\sigma_{4}^{2}=0.01 \sigma_{g}^{2}$, where $\sigma_{g}^{2}$ is the total genetic variance. $\mathbf{u}$ is the vector of random polygenic effects following the normal distribution $\mathbf{u} \sim N\left(0, \mathbf{A} \sigma_{a}^{2}\right)$, in which $\sigma_{a}^{2}$ is the polygenic variance and $\mathbf{A}$ is the pedigree relationship matrix. $\mathbf{Z}$ is an incidence matrix relating phenotypes to the corresponding random polygenic effects. The Markov chain Monte Carlo was run for 20,000 cycles with a burn-in period of 10,000 cycles. Every 10th sample of the remaining 10,000 iterations was saved for estimating SNP effects and the variance components.

The accuracy of genomic prediction was evaluated as the correlation between GEBV and DRP ( $\mathrm{r}_{\mathrm{GEBV}}$,DRP) of the cows in the validation population, corrected with respect to average accuracy (square root of reliability) of DRP - that is, $r_{v}=\frac{r_{G E B V, D R P}}{r_{D R P}}$ (Su et al., 2012b). In addition, $b$ (DRP,GEBV), the regression of DRP on GEBV, was calculated to assess the possible inflation or unbiasedness of predictions.

Linkage disequilibrium between a pair of SNP was measured as $r_{\mathrm{LD}}^{2}$ (Hill and Robertson, 1968) among 54K and imputed HD data sets. The average $r_{\mathrm{LD}}^{2}$ of the imputed HD data set was 0.67 , and 0.25 higher than that 
based on the $54 \mathrm{~K}$ data set across all chromosomes. The average distances of adjacent markers among $54 \mathrm{~K}$ and imputed HD marker panels were 56.7 and $4.6 \mathrm{~kb}$, respectively. For both $54 \mathrm{~K}$ and imputed HD marker panels, the standard deviation of $r_{\mathrm{LD}}^{2}$ among all autosomes was as low as 0.01 and 0.02 , respectively.

Figure 1 and Table 1 show the significant SNP associated with BC, MS, and FL. In total, LM identified $1,403,1,536$, and 1,383 significant SNP associated with $\mathrm{BC}$, MS, and FL, respectively, with only 4 common SNP among them. The significant SNP correspondingly explained $2.7,3.4$, and $2.4 \%$ of genetic variance of 3 traits. The significant SNP were located on the all chromosomes. In addition, only a very small proportion of significant SNP were identified in the $54 \mathrm{~K}$ marker panel (e.g., for BC, 1,312 out of 1,403 significant SNP identified by LM were not included in the $54 \mathrm{~K}$ data).

Three $54 \mathrm{~K}+$ data sets were generated by adding all the significant SNP obtained by LM for each trait into the original 54K SNP chip. Table 1 also shows the accuracy of genomic prediction based on $54 \mathrm{~K}, 54 \mathrm{~K}+$, and HD data. As expected, $54 \mathrm{~K}+$ data generated higher accuracy than $54 \mathrm{~K}$ as extra significant SNP were included. The accuracy was increased $3.9,5.2$, and $2.0 \%$ for $\mathrm{BC}$, MS, and $\mathrm{FL}$, respectively. Interestingly, the improvement of genomic prediction on MS was the largest obtained by BVS; it was consistent with the fact that the largest number of significant SNP were identified and more genetic variance was explained by these significant SNP for MS (Table 1). On the other hand, the HD data did not improve the accuracy of genomic prediction significantly. For BVS, the imputed HD markers yielded 2.5 and $1.7 \%$ higher accuracy than $54 \mathrm{~K}$ data for MS and FL, respectively, but no improvement for BC.

Table 1 also shows that the unbiasedness of genomic prediction was improved by $54 \mathrm{~K}+$ and $\mathrm{HD}$ data. In most situations, $54 \mathrm{~K}+$ data produced the lowest bias. For example, for BC, the regression coefficients $b$ (DRP,GEBV) were $0.535,0.838$, and 0.679 from $54 \mathrm{~K}$, $54 \mathrm{~K}+$, and HD data, respectively. This trend was kept for the other 2 traits.

Our results showed that both the accuracy and the unbiasedness of genomic prediction was increased based on $54 \mathrm{~K}+$ data (see Table 1) compared with the original 54K SNP panel. This is consistent with a previous report (Brøndum et al., 2015) that indicated that the reliability of genomic prediction was increased by up to 4 percentage points for production traits in Nordic Holsteins, up to 3 percentage points for Nordic Reds, and up to 5 percentage points for French Holsteins by adding significant variants based on whole-genome sequence data to the regular 54K SNP chip. Zhang et al.
(2014) also investigated the efficiency of incorporating prior knowledge of complex traits to improve the accuracy of genomic prediction. The results showed that using already-existing GWAS results improved the accuracy of genomic prediction for 2 out of the 3 traits in a dairy cattle data set and for 9 out of the 11 traits in a rice diversity data set (Zhang et al., 2014).

The present study also shows that the imputed HD markers yielded 2.5 and $1.7 \%$ higher accuracy of genomic prediction than $54 \mathrm{~K}$ data for MS and FL but no improvement for BC. Numerous recent studies also indicated that marker density does not have a significant effect on prediction ability compared with the performance of 54K data (Harris and Johnson, 2010; VanRaden et al., 2011; Su et al., 2012a; VanRaden et al., 2013). One possible reason is that the advantage of increasing pairwise LD by HD markers might be counteracted by increasing the number of unknown parameters to be estimated, as pointed out by $\mathrm{Su}$ et al. (2012a). Another reason is that the HD marker genotypes were not real marker genotypes using HD chips but rather imputed ones. The investigation of imputed BovineLD to $54 \mathrm{~K}$ data showed that each $1 \%$ of imputation allele error rate resulted in a loss of reliability of 1.3 percentage points (Dassonneville et al., 2011). However, it should be noted that the imputed HD data generated lower bias than the $54 \mathrm{~K}$ data even though the improvement in accuracy was small.

In China, genomic selection in Chinese Holstein cattle has been carried out since 2009 and has been implemented in practical breeding since 2012. A reference population has been built and continuously updated. Unlike in other countries, where thousands of progeny tested bulls with highly reliable EBV are used to form a national reference population, in Chinese Holstein cattle the reference population for genomic selection mainly consists of cows. Ding et al. (2013) reported that the genomic selection using cows was feasible, whereas the accuracy was still lower compared with using progeny-tested bulls as a reference population. Li et al. (2014) investigated whether the accuracy of genomic prediction of milk production traits in Chinese Holsteins can be improved by using the single-step approach. In the genomic evaluation of Chinese Holsteins, milk production traits, health traits (SCS), and body conformation traits were mainly considered. A reference population with sufficient size is essential in genomic selection; compared with milk production traits and health traits, the amount of both phenotypic records and genotyped individuals of body conformation traits are smaller. Our results showed that the accuracy of genomic prediction on body conformation traits could be improved using the markers derived from HD marker 

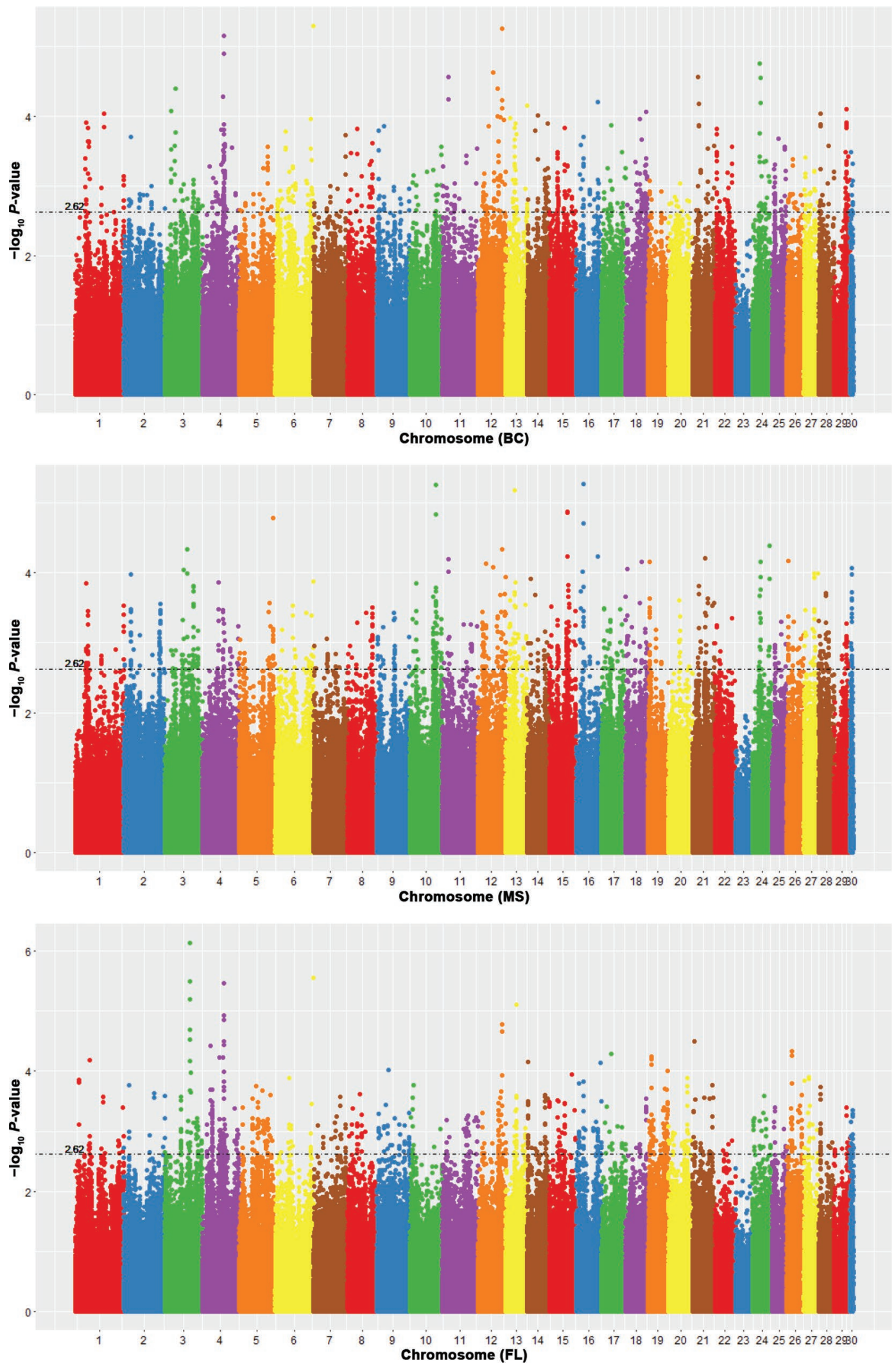

Figure 1. Manhattan plots of genome-wide association study for 3 body conformation traits: conformation final score (BC), mammary system (MS), and feet and legs (FL). Color version available online. 
Table 1. Summary of significant SNP associated with 3 body conformation traits identified by linear mixed (LM) model based on imputed high-density (HD) marker panel and accuracy and unbiasedness (in parentheses) of genomic prediction obtained by Bayesian variable selection (BVS) model based on $54 \mathrm{~K}, 54 \mathrm{~K}+$, and imputed HD data sets

\begin{tabular}{|c|c|c|c|c|c|}
\hline Trait & LM & $\begin{array}{l}\text { Explained genetic } \\
\text { variance (\%) }\end{array}$ & \multicolumn{3}{|c|}{ BVS } \\
\hline $\begin{array}{l}\text { Conformation final score } \\
\text { Mammary system } \\
\text { Feet and legs }\end{array}$ & $\begin{array}{l}1,403\left(1,312^{1}\right) \\
1,536\left(1,428^{1}\right) \\
1,383\left(1,302^{1}\right)\end{array}$ & $\begin{array}{l}2.7 \\
3.4 \\
2.4\end{array}$ & $\begin{array}{l}0.488(0.535) \\
0.297(0.357) \\
0.513(0.536)\end{array}$ & $\begin{array}{l}0.527(0.838) \\
0.349(0.610) \\
0.533(0.716)\end{array}$ & $\begin{array}{l}0.488(0.679) \\
0.322(0.537) \\
0.530(0.689)\end{array}$ \\
\hline
\end{tabular}

${ }^{1}$ Number of significant SNP identified by LM model not included in the $54 \mathrm{~K}$ marker panel.

panels via GWAS. It would also be attractive to other traits and be useful for practical genomic selection in Chinese Holsteins.

\section{ACKNOWLEDGMENTS}

This work was supported by National Natural Science Foundation of China (31272418), the grants from the earmarked fund for China Agriculture Research System (CARS-36), National Dairy Industry System in Beijing Team, Program for Changjiang Scholar and Innovation Research Team in University (Grant No. IRT_15R621). We thank Dairy Association of China for supplying the official EBV and Beijing Dairy Cattle Center for providing blood and semen samples.

\section{REFERENCES}

Brøndum, R. F., G. Su, L. Janss, G. Sahana, B. Guldbrandtsen, D. Boichard, and M. S. Lund. 2015. Quantitative trait loci markers derived from whole genome sequence data increases the reliability of genomic prediction. J. Dairy Sci. 98:4107-4116.

Dassonneville, R., R. F. Brondum, T. Druet, S. Fritz, F. Guillaume, B. Guldbrandtsen, M. S. Lund, V. Ducrocq, and G. Su. 2011. Effect of imputing markers from a low-density chip on the reliability of genomic breeding values in Holstein populations. J. Dairy Sci. 94:3679-3686.

Ding, X., Z. Zhang, X. Li, S. Wang, X. Wu, D. Sun, Y. Yu, J. Liu, Y. Wang, Y. Zhang, S. Zhang, Y. Zhang, and Q. Zhang. 2013. Accuracy of genomic prediction for milk production traits in the Chinese Holstein population using a reference population consisting of cows. J. Dairy Sci. 96:5315-5323.

Erbe, M., B. J. Hayes, L. K. Matukumalli, S. Goswami, P. J. Bowman, C. M. Reich, B. A. Mason, and M. E. Goddard. 2012. Improving accuracy of genomic predictions within and between dairy cattle breeds with imputed high-density single nucleotide polymorphism panels. J. Dairy Sci. 95:4114-4129.

Goddard, M. E., and B. J. Hayes. 2009. Mapping genes for complex traits in domestic animals and their use in breeding programmes. Nat. Rev. Genet. 10:381-391.

Harris, B. L., and D. L. Johnson. 2010. The impact of high density SNP chips on genomic evaluation in dairy cattle. Interbull Bull. 42:40-43.

Hill, W. G., and A. Robertson. 1968. Linkage disequilibrium in finite populations. Theor. Appl. Genet. 38:226-231.

Jairath, L., J. C. M. Dekkers, L. R. Schaeffer, Z. Liu, E. B. Burnside, and B. Kolstad. 1998. Genetic evaluation for herd life in Canada. J. Dairy Sci. 81:550-562.
Li, X., S. Wang, J. Huang, L. Y. Li, Q. Zhang, and X. D. Ding. 2014 Improving the accuracy of genomic prediction in Chinese Holstein cattle by using one-step blending. Genet. Sel. Evol. 46:66.

Meredith, B. K., D. P. Berry, F. Kearney, E. K. Finlay, A. G. Fahey, D. G. Bradley, and D. J. Lynn. 2013. A genome-wide association study for somatic cell score using the Illumina high-density bovine beadchip identifies several novel QTL potentially related to mastitis susceptibility. Front. Genet. 4:229.

Meuwissen, T. H. E., B. J. Hayes, and M. E. Goddard. 2001. Prediction of total genetic value using genome-wide dense marker maps. Genetics 157:1819-1829.

Ober, U., J. F. Ayroles, E. A. Stone, S. Richards, D. H. Zhu, R. A Gibbs, C. Stricker, D. Gianola, M. Schlather, T. F. C. Mackay, and H. Simianer. 2012. Using whole-genome sequence data to predict quantitative trait phenotypes in Drosophila melanogaster. PLoS Genet. 8.

Sahana, G., B. Guldbrandtsen, B. Thomsen, L. E. Holm, F. Panitz, R. F. Brondum, C. Bendixen, and M. S. Lund. 2014. Genomewide association study using high-density single nucleotide polymorphism arrays and whole-genome sequences for clinical mastitis traits in dairy cattle. J. Dairy Sci. 97:7258-7275.

Sargolzaei, M., J. Chesnais, and F. Schenkel. 2011. FImpute-An efficient imputation algorithm for dairy cattle populations. J. Dairy Sci. 94:421.

Spencer, C. C., Z. Su, P. Donnelly, and J. Marchini. 2009. Designing genome-wide association studies: Sample size, power, imputation, and the choice of genotyping chip. PLoS Genet. 5:e1000477.

$\mathrm{Su}$, G., R. F. Brondum, P. Ma, B. Guldbrandtsen, G. R. Aamand, and M. S. Lund. 2012a. Comparison of genomic predictions using medium-density (similar to 54,000) and high-density (similar to $777,000)$ single nucleotide polymorphism marker panels in Nordic Holstein and Red Dairy cattle populations. J. Dairy Sci. 95:46574665 .

Su, G., P. Madsen, U. S. Nielsen, E. A. Mantysaari, G. P. Aamand, O. F. Christensen, and M. S. Lund. 2012b. Genomic prediction for Nordic Red cattle using one-step and selection index blending. J. Dairy Sci. 95:909-917.

VanRaden, P. M. 2008. Efficient methods to compute genomic predictions. J. Dairy Sci. 91:4414-4423.

VanRaden, P. M., D. J. Null, M. Sargolzaei, G. R. Wiggans, M. E. Tooker, J. B. Cole, T. S. Sonstegard, E. E. Connor, M. Winters, J. B. C. H. M. van Kaam, A. Valentini, B. J. Van Doormaal, M. A. Faust, and G. A. Doak. 2013. Genomic imputation and evaluation using high-density Holstein genotypes. J. Dairy Sci. 96:668-678.

VanRaden, P. M., J. R. O'Connell, G. R. Wiggans, and K. A. Weigel. 2011. Genomic evaluations with many more genotypes. Genet. Sel. Evol. 43:10

Yang, J., S. H. Lee, M. E. Goddard, and P. M. Visscher. 2011. GCTA: A tool for genome-wide complex trait analysis. Am. J. Hum. Genet. $88: 76-82$.

Zhang, Z., U. Ober, M. Erbe, H. Zhang, N. Gao, J. L. He, J. Q. Li, and H. Simianer. 2014. Improving the accuracy of whole genome prediction for complex traits using the results of genome wide association studies. PLoS One 9:e93017. 